The Astrophysical Journal, 279:136-143, 1984 April 1

(c) 1984. The American Astronomical Society. All rights reserved. Printed in U.S.A.

\title{
THE RADIAL DISTRIBUTION OF GALACTIC GAMMA-RAYS. I. EMISSIVITY AND EXTENT IN THE OUTER GALAXY
}

\author{
J. B. G. M. Bloemen, ${ }^{1,2}$ L. Blitz, ${ }^{1,3}$ AND W. HermSEN ${ }^{2}$ \\ Received 1983 May 19; accepted 1983 September 7
}

\begin{abstract}
A method is presented by which the radial distribution of galactic high-energy $\gamma$-rays $(70 \mathrm{MeV}-5 \mathrm{GeV})$ can be determined. It is shown that the $\gamma$-ray intensity for $R>R_{\odot}$ depends on $N(\mathrm{H} \mathrm{I})$ alone to within the uncertainties of the method. The kinematics of the $\mathrm{H}$ I are used to show that galactic $\gamma$-rays, and consequently cosmic-ray particles, are present in significant quantities to galactocentric distances of approximately $17 \mathrm{kpc}$. The $\gamma$-ray emissivity $(70 \mathrm{MeV}-5 \mathrm{GeV})$ at $R>R_{\odot}$ is found to be $(2.12 \pm 0.07) \times 10^{-26}$ photons $\mathrm{H} \mathrm{atom}^{-1} \mathrm{~s}^{-1} \mathrm{sr}^{-1}$, within $15 \%$ of the local value, and the $\gamma$-ray luminosity $(>100 \mathrm{MeV})$ of the Milky Way is $(1.3-2.5) \times 10^{42}$ photons s${ }^{-1}\left([1.6-3.2] \times 10^{39}\right.$ ergs s$\left.^{-1}\right)$. The $\mathrm{H}_{2}$ mass at $R>R_{\odot}$ is found to be $<3 \times 10^{8} M_{\odot}$.

Subject headings: cosmic rays: general — galaxies: Milky Way - gamma rays: general interstellar: matter - interstellar: molecules
\end{abstract}

\section{INTRODUCTION}

The diffuse component of galactic high-energy $\gamma$-rays $(\gtrsim 50 \mathrm{MeV}$ ) has long been thought to be mainly the result of interactions between cosmic rays and the nuclei of interstellar gas (e.g., Fazio 1967 and Stecker 1971). Using the COS B data, Lebrun et al. (1982) and Strong et al. (1982) have shown that locally ( $\lesssim 1 \mathrm{kpc}$ ), the $\gamma$-ray intensity is correlated with the total gas column density along the same line of sight. If this correlation holds for the Galaxy as a whole, it is then possible to use the kinematics of the gas coupled with the rotation curve as a tracer for the locations of $\gamma$-ray production. This information can then be used to determine the radial distribution of the diffuse $\gamma$-rays.

In this paper, we show that beyond the solar circle, the $\gamma$-ray intensity is proportional to the $\mathrm{H} \mathrm{I}$ column density alone within the uncertainties, and that the emissivity in the $70 \mathrm{MeV}-5 \mathrm{GeV}$ energy range is only about $15 \%$ lower than the local value. We then examine the radial distribution of the $\mathrm{H}_{\mathrm{I}}$ with which the $\gamma$-rays are correlated and show that a significant fraction of the $\gamma$-ray emission originates at large galactocentric distances, which implies that cosmic rays must be abundant in the outer Galaxy. The results are shown to warrant a more detailed analysis which will be the subject of a forthcoming paper (Bloemen et al. 1984).

\section{THE CONTRIBUTION OF $\mathrm{H}_{2}$ AT $R>R_{\odot}$}

Using galaxy counts and $\operatorname{COS} B \gamma$-ray data in the latitude range $10^{\circ}<|b|<20^{\circ}$, Lebrun et al. (1982) and Strong et al. (1982) have shown that the $\gamma$-ray intensity is well correlated with the total column density of hydrogen nuclei $\left[N\left(\mathrm{H}_{\mathrm{I}}\right)+2 N\left(\mathrm{H}_{2}\right)\right]$ in the solar vicinity. The derived local emissivity $\left(\epsilon_{\gamma}=q_{\gamma} / 4 \pi\right)$ in the energy range $70 \mathrm{MeV}-5 \mathrm{GeV}$ is $2.5 \times 10^{-26}$ photons $\mathrm{H}$ atom ${ }^{-1} \mathrm{~s}^{-1} \mathrm{sr}^{-1}$ with an uncertainty (which is mainly systematic) of about $25 \%$ (Strong et al.

\footnotetext{
${ }^{1}$ Sterrewacht, Huygens Laboratorium, Leiden, The Netherlands.

${ }^{2}$ Laboratory for Space Research Leiden, Leiden, The Netherlands.

${ }^{3}$ University of Maryland.
}

1982). Since $I_{\gamma}=\epsilon_{\gamma}\left[N\left(\mathrm{H} \mathrm{I}_{\mathrm{I}}\right)+2 N\left(\mathrm{H}_{2}\right)\right]+I_{B}$, where $I_{\gamma}$ is the observed $\operatorname{COS} B \gamma$-ray intensity and $I_{B}$ is the underlying isotropic background level, it is in principle possible to determine $\epsilon_{\gamma}$ in regions within $10^{\circ}$ of the galactic plane from $21 \mathrm{~cm}$ and $\mathrm{CO}$ observations. There is, however, no complete $\mathrm{CO}$ survey of the galactic plane, and in any event, the derived $\mathrm{H}_{2}$ column densities are generally thought to be uncertain by about a factor of 3 .

Beyond the solar circle, however, the $\mathrm{H}_{2}$ mass appears to be small compared to the $\mathrm{H}_{\mathrm{I}}$ mass. Estimates of the $\mathrm{H}_{2}$ mass at $R>R_{\odot}$ have been given by Kutner and Mead (1981) to be $3 \times 10^{8} M_{\odot}$ (with a possible upward correction of about a factor of 2 for scale height effects). Solomon, Stark, and Sanders (1983) have argued that the number of clouds between $R=11 \mathrm{kpc}$ and $R=15 \mathrm{kpc}$ is $12 \%$ of the number of clouds between $R=3 \mathrm{kpc}$ and $R=9 \mathrm{kpc}$, with very few clouds beyond $R=15 \mathrm{kpc}$. Using Sanders's (1982) estimate of $\mathrm{H}_{2}$ mass of $2.7 \times 10^{9} \quad M_{\odot}$ between $R=2 \mathrm{kpc}$ and $R=10 \mathrm{kpc}$, one derives an approximate $\mathrm{H}_{2}$ mass of $3 \times 10^{8} M_{\odot}$ at $R>R_{\odot}$. For the $\mathrm{H} \mathrm{I}$, Henderson, Jackson, and Kerr (1982) derive a total H I mass for the Milky Way of $4.8 \times 10^{9} M_{\odot}$, of which $80 \%$ lies beyond $R=R_{\odot}$. Thus for a typical line of sight in the second and third galactic quadrants, one expects $N\left(\mathrm{H}_{2}\right) / N\left(\mathrm{H}_{\mathrm{I}}\right) \approx 0.08-0.15$. On average, it is therefore expected that it is possible to ignore the molecular gas in obtaining $\gamma$-ray emissivities with uncertainties due to the $\mathrm{H}_{2}$ contribution of $\sim 10 \%$. However, because of the large uncertainties in the $\mathrm{CO} / \mathrm{H}_{2}$ conversion, and the disagreements among $\mathrm{CO}$ observers regarding the outer Galaxy results, we have made an independent check of the hypothesis that the $\mathrm{H}_{2}$ can be ignored.

If the $\mathrm{H}_{2}$ contributes significantly to the $\gamma$-ray emissivity, there should be a measurable difference between the locally determined emissivity (which includes the $\mathrm{H}_{2}$ contribution) and the value found in the second and third quadrants determined from the $21 \mathrm{~cm}$ observations alone. Furthermore, the $\mathrm{CO}$ scale height is significantly smaller than the $\mathrm{H}$ I scale height outside the solar circle (Fich and Blitz 1983; Kulkarni, Blitz, and Heiles 1982). The $\gamma$-ray emissivity at $R>R_{\odot}$ should therefore show a latitude dependence, if the $\mathbf{H}_{2}$ 
contribution is significant, and if the $\mathrm{H}_{\mathrm{I}}$ alone is used to determine the $\gamma$-ray emissivity.

To determine the $\gamma$-ray emissivity at $R>R_{\odot}$, we use the $\operatorname{COS} B$ data in the longitude range covered by the Weaver and Williams (1973) $\mathrm{H}$ I survey in the second and third quadrants. Gamma-ray intensity maps have been derived from the $\operatorname{COS} B$ data base described by Mayer-Hasselwander et al. (1982), supplemented by later observations. The decrease in instrument sensitivity as a function of its lifetime in orbit has been taken into account by comparison of measured intensities from many selected regions along the galactic plane which were viewed at different epochs. An $E^{-2}$ input spectrum, as derived for the diffuse emission in the solar neighbourhood (see, e.g., Lebrun et al. 1982), has been assumed when taking into account the energy dependence of the instrument response for the determination of the $\gamma$-ray intensities. The correction for the isotropic background emission (mainly instrumental) is left as a free parameter.
Those regions where contamination from seven known pointlike $\gamma$-ray sources (Swanenburg et al. 1981) and known concentrations of molecules between $l=107^{\circ}$ and $l=113^{\circ}$ might affect the analysis are excluded. We analyse the remaining data in the longitude range $l=95^{\circ}-245^{\circ}$ and the latitude range $|b|<10^{\circ}$.

The $\mathrm{H}_{\text {I }}$ survey data are corrected to obtain the brightness temperature $T_{b}$ (Williams 1973), and an optical depth correction is made assuming that the spin temperature is everywhere $125 \mathrm{~K}$. The resulting data are convolved with the $\operatorname{COS} B$ point spread function (Hermsen 1980), and the $\mathrm{H}$ I survey of Heiles and Habing (1974) is used to obtain the contribution to the convolved maps from $|b|>10^{\circ}$. The $\mathrm{H}$ I data are binned in $4^{\circ} \times 2^{\circ}(l \times b)$ bins and compared to the $\operatorname{COS} B$ data along the same lines of sight.

The data are analyzed by comparing $I_{\gamma}$ and $N(\mathrm{H} \mathrm{I})$ bin by bin in the energy ranges $70 \mathrm{MeV}-5 \mathrm{GeV}$ and $150 \mathrm{MeV}-$ $5 \mathrm{GeV}$. The results are presented in Figures $1 a$ and $1 b$.
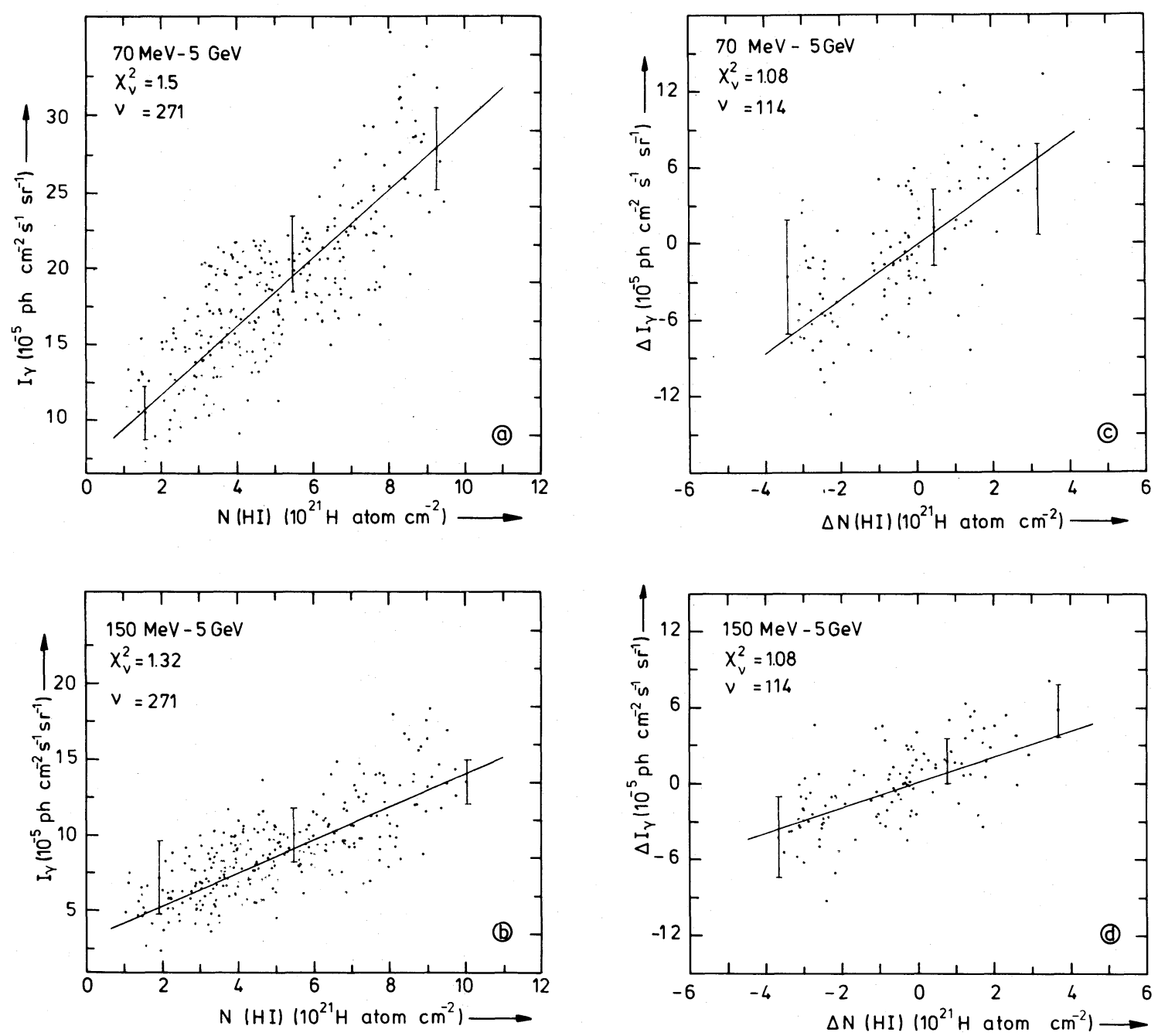

FIG. 1.-Comparison of $\gamma$-ray intensities and $\mathrm{H}$ I column densities in the second and third galactic quadrants for two energy ranges. Plots $a$ and $b$ show $I_{\gamma}$ vs. $N\left(\mathrm{H}_{\mathrm{I}}\right)$ in $4^{\circ} \times 2^{\circ}(l \times b)$ bins. Plots $c$ and $d$ show $\Delta I_{\gamma}$ vs. $\Delta N(\mathrm{H} \mathrm{I})$ [for definition of $\Delta I_{\gamma}$ and $\Delta N(\mathrm{H} \mathrm{I})$, see text] in the same bin sizes. The statistical error bars given are characteristic for most points. The solid lines indicate the least squares fits to the data points, and their slopes correspond to the $\gamma$-ray emissivities in Table 1 . 


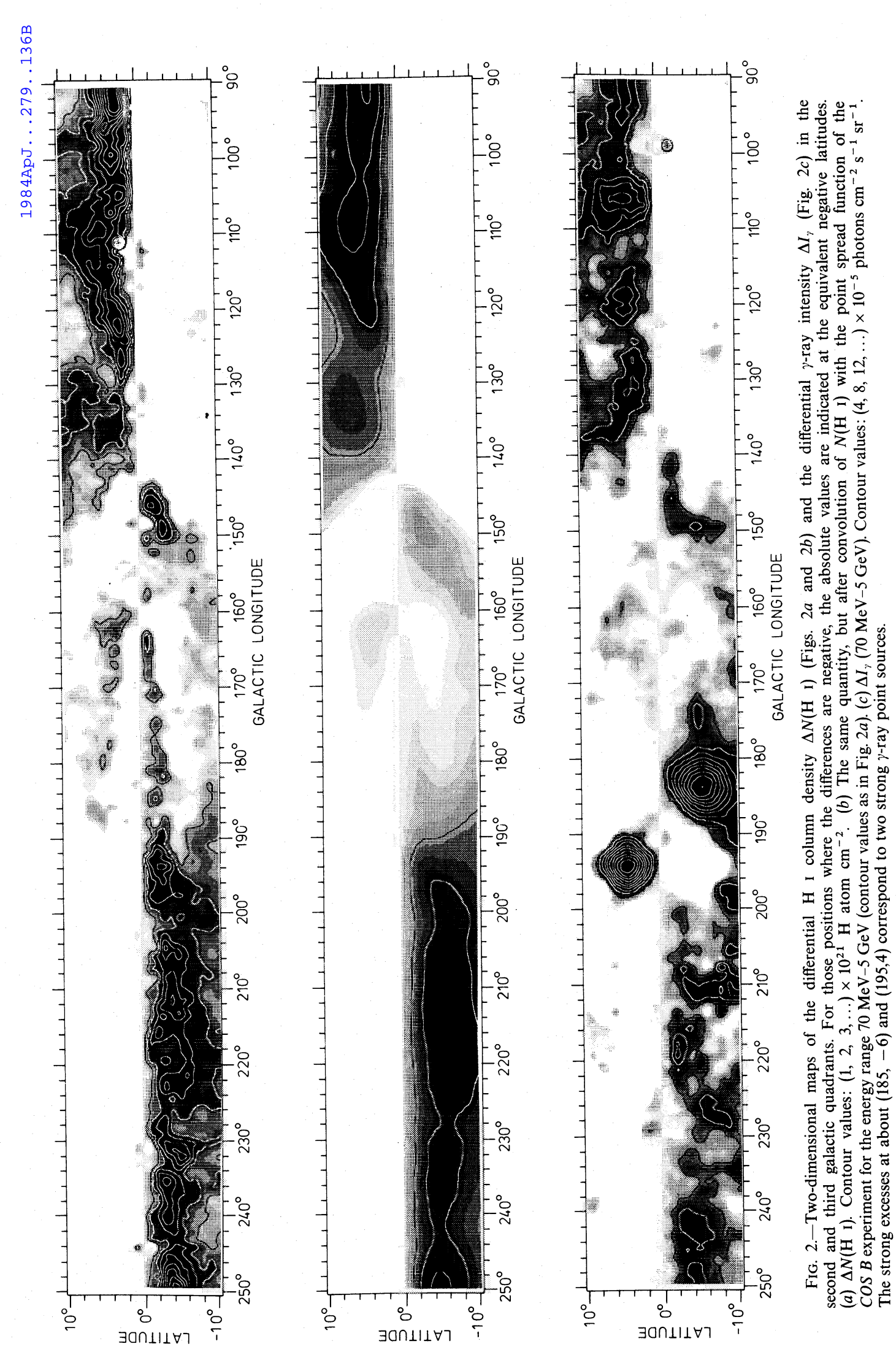


TABLE 1

Gamma-Ray Emissivity $\epsilon_{\gamma}$ In the Second and Third Galactic Quadrants and $|b|<10^{\circ}$, as Determined FROM THE COMPARISON OF $I_{\gamma}$ WITH $N(\mathrm{H}$ I $)$ AND FROM THE COMPARISON OF $\Delta I_{\gamma}$ WITH $\Delta N(\mathrm{H}$ I $)$

\begin{tabular}{|c|c|c|}
\hline & $\begin{array}{c}\epsilon_{y}(150 \mathrm{MeV}-5 \mathrm{GeV}) \\
\left(10^{-26} \text { photons } \mathrm{H} \text { atom }{ }^{-1} \mathrm{~s}^{-1} \mathrm{sr}^{-1}\right)\end{array}$ & 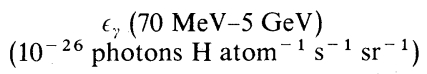 \\
\hline From $I_{\gamma}$ vs. $N\left(\mathrm{H}_{\mathrm{I}}\right) \ldots$ & $1.06 \pm 0.05$ & $2.12 \pm 0.07$ \\
\hline From $\Delta I$, vs. $\Delta N(\mathrm{H} \mathrm{I})$. & $1.01 \pm 0.11$ & $2.11 \pm 0.19$ \\
\hline 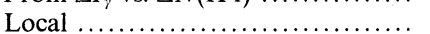 & $1.12^{\mathrm{a}-}$ & $2.52^{\mathrm{a}}$ \\
\hline
\end{tabular}

Note.-The local $\gamma$-ray emissivities are those given by Strong et al. 1982.

${ }^{a}$ Strong et al. 1982 mention that these values have systematic uncertainties of about $25 \%$ (for comparison with our outer Galaxy emissivities, see text).

A good correlation is evident in the figures. The slope of the least squares fit defines $\epsilon_{\gamma}$ in both energy ranges, the values of which are given in Table 1 . The offset of the fit defines the isotropic background emission in both energy ranges. The background levels resulting from the present analysis are consistent with the values determined by Strong et al. (1982) (using the same $\operatorname{COS} B$ data base).

Since the background level entered the analysis as a free parameter, one would like to verify that the derived emissivities are independent of the resulting background. This may be done by subtracting the data at negative latitudes from those at equivalent positive latitudes to provide a comparison between $\Delta I_{\gamma}$ and $\Delta N(\mathrm{H} \mathrm{I})$, where $\Delta$ is the excess emission at positive latitudes. This analysis is made possible because of the large-scale warp of the hydrogen layer and has the additional advantage (discussed in $\S$ III) that the gas contributing to $\Delta N\left(\mathrm{H} \mathrm{I}_{\mathrm{I}}\right)$ is, on average, at a larger distance than that contributing to $N(\mathrm{H} \mathrm{I})$. Using $\Delta I_{\gamma}$ and $\Delta N\left(\mathrm{H}_{\mathrm{I}} \mathrm{I}\right)$ therefore provides a measure of $\epsilon_{\gamma}$ at relatively larger galactocentric distances. Plots of a bin by bin comparison of $\Delta I_{\gamma}$ and $\Delta N(\mathrm{H} \mathrm{I})$ are given in Figure $1 c$ and $1 d$. The resulting values of $\epsilon_{\gamma}$ are also given in Table 1. Figure 2 presents the two-dimensional maps of $\Delta I_{\gamma}$ and $\Delta N(\mathrm{H} \mathrm{I})$, showing the good detailed correlation between these quantities.

The values in Table 1 show that the emissivities determined from $N(\mathrm{H} \mathrm{I})$ and $\Delta N(\mathrm{H} \mathrm{I})$ are in good agreement in both energy ranges. Therefore the background level determined from the analysis of $N(\mathrm{H} \mathrm{I})$ is reliable. Because Strong et al. (1982) and the present analysis treat the $\operatorname{COS} B$ data in the same way, and because the background levels are found to be consistent in both analyses, a direct comparison of our results can be made to the local emissivity values. In the systematic uncertainties $(\sim 25 \%)$ mentioned by Strong et al. in determining the local values, about $15 \%$ were due to uncertainties in the derived background levels. The emissivity in the $150 \mathrm{MeV}-5 \mathrm{GeV}$ range is equal to the local value of $1.1 \times 10^{-26}$ photons $\mathrm{H}$ atom ${ }^{-1} \mathrm{~s}^{-1} \mathrm{sr}^{-1}$ given by Strong et al. In the energy range of $70 \mathrm{MeV}-5 \mathrm{GeV}$ the emissivity is $\sim 15 \%$ lower than the local value of $2.5 \times 10^{-26}$ photons $\mathrm{H}$ atom ${ }^{-1} \mathrm{~s}^{-1} \mathrm{sr}^{-1}$. Because of remaining uncertainties of $\sim 10 \%$ in determining the local value, it is unclear whether the decrease in the $70 \mathrm{MeV}-5 \mathrm{GeV}$ emissivity is significant. If it is significant, the decrease is mainly due to a lowered (by $\sim 30 \%$ ) emissivity in the $70-150 \mathrm{MeV}$ energy range. A detailed analysis of this point will be the subject of the second paper of this series. In any event, the values in
Table 1 show that the emissivities determined from the $\mathrm{H}$ I data are equal to or less than the local values, thus justifying the assumption that, on average, the contribution from $\mathrm{H}_{2}$ can be ignored in the outer Galaxy. On the other hand, a large $\gamma$-ray emissivity gradient would mask a dependence on $\mathrm{H}_{2}$, but this can be checked by an analysis of the latitude dependence of the emissivity.

To determine the latitude dependence, the data are analyzed as above for each latitude and the results are plotted in Figure 3. As before, the results are plotted separately for $I_{\gamma}$ and $\Delta I_{\gamma}$ as well as for the two energy ranges we have analyzed. Figure 3 shows that there is no significant increase in the $\gamma$-ray emissivity at low latitudes. We therefore conclude that to within $7 \%(2 \sigma ;$ Fig. $3 a)$, molecular hydrogen can be ignored as a source of $\gamma$-rays in the outer Galaxy. Thus, on average, the $\gamma$-ray intensities in the outer Galaxy are shown to be dependent on $N\left(\mathrm{H} \mathrm{I}_{\mathrm{I}}\right)$ only. Since most of the $\mathrm{H}_{2}$ in the outer Galaxy at $R>11 \mathrm{kpc}$ is within $\pm 4^{\circ}$ of the plane (see the catalog of Blitz, Fich, and Stark 1982), and $\epsilon_{\gamma}$ does not change at higher latitudes, the $\mathrm{H}_{2}$ mass at $\mathrm{R}>R_{\odot}$ cannot be more than $\sim 3 \times 10^{8} M_{\odot}$.

In principle, the emissivities given in Table 1 should be corrected for a contribution from inverse Compton $\gamma$-rays and the small contribution from $\mathrm{H}_{2}$. Kniffen and Fichtel (1981) and Sacher and Schönfelder (1983) have shown that although the inverse Compton effect may be significant in the inner Galaxy, it is small $(\lesssim 10 \%)$ for $R>R_{\odot}$ compared to the contribution from the cosmic-ray-matter interactions. For the contribution of $\gamma$-rays originating from $\mathrm{H}_{2}$, a limit of $\sim 7 \%$ has been given above for the outer Galaxy. Inclusion of these effects will negligibly change the tabulated emissivities because the low-energy photon field and the $\mathrm{H}_{2}$ gas would have to be spatially well correlated with the $\mathrm{H}$ I. Since the correlations are probably fairly poor, we expect that the lower bound of the values given in Table 1 will only be lowered by a few percent.

\section{THE RADIAL DISTRIBUTION}

The good correlation between the $\gamma$-ray intensities and the $\mathrm{H}$ I column densities in the outer Galaxy, with the average $\epsilon_{y}$ along the line of sight close to the local value, indicates that the $\gamma$-ray distribution must be strongly coupled to the $\mathrm{H}$ I distribution (at least for $\gamma$-rays in the $150 \mathrm{MeV}-5 \mathrm{GeV}$ range). The velocity information available from the $21 \mathrm{~cm}$ line can be used to determine the radial $\mathrm{H}$ I distribution, which, because of the near constancy of $\epsilon_{i}$ will be very 



Fig. 3.-Gamma-ray emissivities $\epsilon_{i}$ as a function of galactic latitude in the second and third galactic quadrants for the two energy ranges. Plots $a$ and $b$ show the results obtained by a comparison of $I_{2}$ and $N(\mathrm{H} \mathrm{I})$. Plots $c$ and $d$ present the results from the comparison of $\Delta I_{y}$, and $\Delta N(\mathrm{H} \mathrm{I})$. The dashed lines indicate the $\gamma$-ray emissivities for the total latitude range $\left(|b|<10^{\circ}\right)$ given in Table 1 .

similar to the radial $\gamma$-ray distribution. We use the rotation curve of the outer Galaxy of Blitz, Fich, and Stark (1980) as modified by Kulkarni, Blitz, and Heiles (1982) to determine distances beyond the solar circle.

To establish the radial distribution of the $\mathrm{H}$, we ask within what distance does $25 \%, 50 \%$ and $75 \%$ of the $\mathrm{H}$ I emission lie, and produce maps of these distances for both $N(\mathrm{H} \mathrm{I})$ and $\Delta N(\mathrm{H} \mathrm{I})$. These maps are presented as Figures 4 and 5. The longitude range $165^{\circ}-195^{\circ}$ is excluded because one cannot obtain reliable kinematic distances.

A number of conclusions may be drawn from these maps regarding the $\mathrm{H}$ I distribution and consequently the $\gamma$-ray distribution. (1) The maps show that the distances are strongly latitude dependent which implies that emission near the plane tends to sample gas and $\gamma$-rays at larger distances than does the higher latitude emission, a result which has long been known. (2) $\Delta N\left(\mathrm{H}_{\mathrm{I}}\right)$ samples gas and $\gamma$-rays at larger distances than $N(\mathrm{HI})$ at a given $l, b$. The reason is that the maps of $\Delta N(\mathrm{H} \mathrm{I})$ subtract the component of the relatively local distribution that is symmetric with respect to the galactic plane. The warp in the $\mathrm{H}$ I plane becomes more pronounced at larger galactocentric distances (see, e.g., Kulkarni, Blitz, and Heiles 1982); thus the asymmetric emission component at large distances is preferentially mapped by $\Delta N\left(\begin{array}{ll}\mathrm{H} & \mathrm{I}\end{array}\right)$. (3) About $\frac{1}{4}$ of the $\mathrm{H}$ I and $\gamma$-ray emission is found to be emitted from regions between $R \approx 14 \mathrm{kpc}$ and $R \approx 17 \mathrm{kpc}$ near the galactic plane. The diffuse component of the galactic high-energy $\gamma$-ray emission therefore extends to very large galactocentric distances. Since $\epsilon_{\gamma}$ is nearly constant and nearly equal to the local value, the cosmic-ray density must be large and nearly equal to the local value at distances as large as 14-17 kpc from the galactic center. In a recent study Schlosser and Feitzinger (1983) claim incorrectly that the major part of the $\gamma$-ray emission in the second and third quadrants is produced in the local spiral arm (for remarks on their analysis see Bloemen, Blitz, and Hermsen 1983). (4) The maps show a longitude dependence of the distance from which a given percentage of the emission originates in the sense that larger distances tend to occur at larger longitudes. This effect is caused by the spiral structure of the outer Milky Way which is best seen in the H I surface density plots of Kulkarni, Blitz, and Heiles (1982).

With regard to point (3) above, it is necessary to keep in mind that the remaining uncertainties of $\sim 10 \%$ in the local emissivities of Strong et al. (1982) lead to uncertainties in the ratio of outer-Galaxy-to-local emissivities of $\sim 10 \%$. These ratios are $0.95 \pm 0.10$ for the $150 \mathrm{MeV}-5 \mathrm{GeV}$ range and $0.84 \pm 0.09$ for the $70 \mathrm{MeV}-5 \mathrm{GeV}$ range. In the most extreme case, one finds an average emissivity in the outer Galaxy which is $75 \%$ of the local value. We cannot exclude this possibility with the present analysis; such a lowered emissivity would imply that the distances within which $25 \%$, $50 \%$, and $75 \%$ of the $\gamma$-ray production is located are somewhat overestimated in the galactic plane. However, we point out that the close correlation between the $\gamma$-ray intensities and $\mathrm{H}$ I column densities shown in Figures 1 and 2 requires that any emissivity decrease be monotonic and smooth, as opposed to abruptly cutting off at some distance $R$. This can only be true if the production rate of $\gamma$-rays is still substantial at large $R$, validating the conclusion that the production of $\gamma$-rays is significant at distances of $14-17 \mathrm{kpc}$ from the galactic center.

\section{THE GAMMA-RAY LUMINOSITY OF THE MILKY WAY}

The diffuse component of the galactic $\gamma$-ray luminosity can be determined from mass estimates of the gas content of the Galaxy and the $\gamma$-ray emissivity. Since a small decrease 


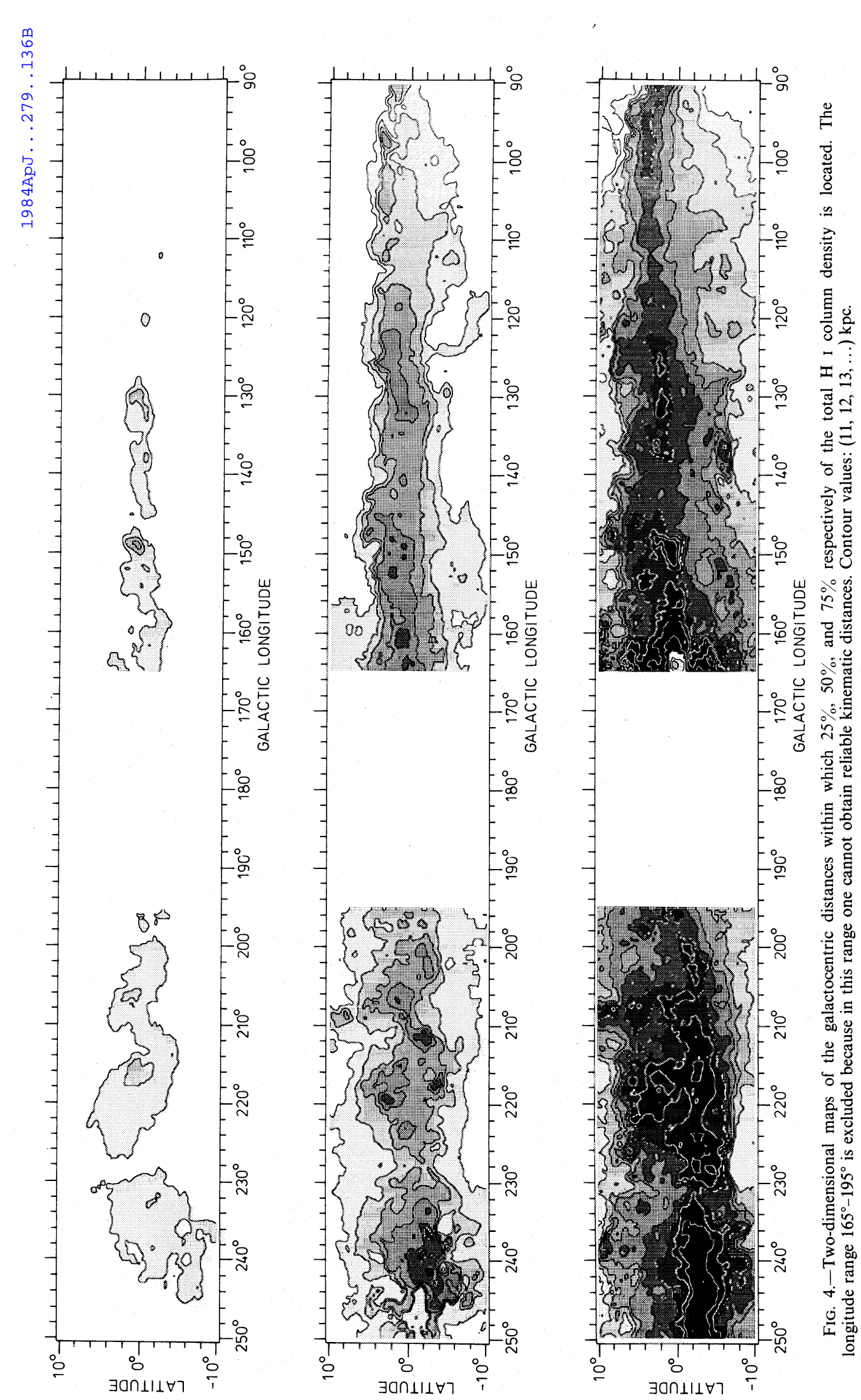




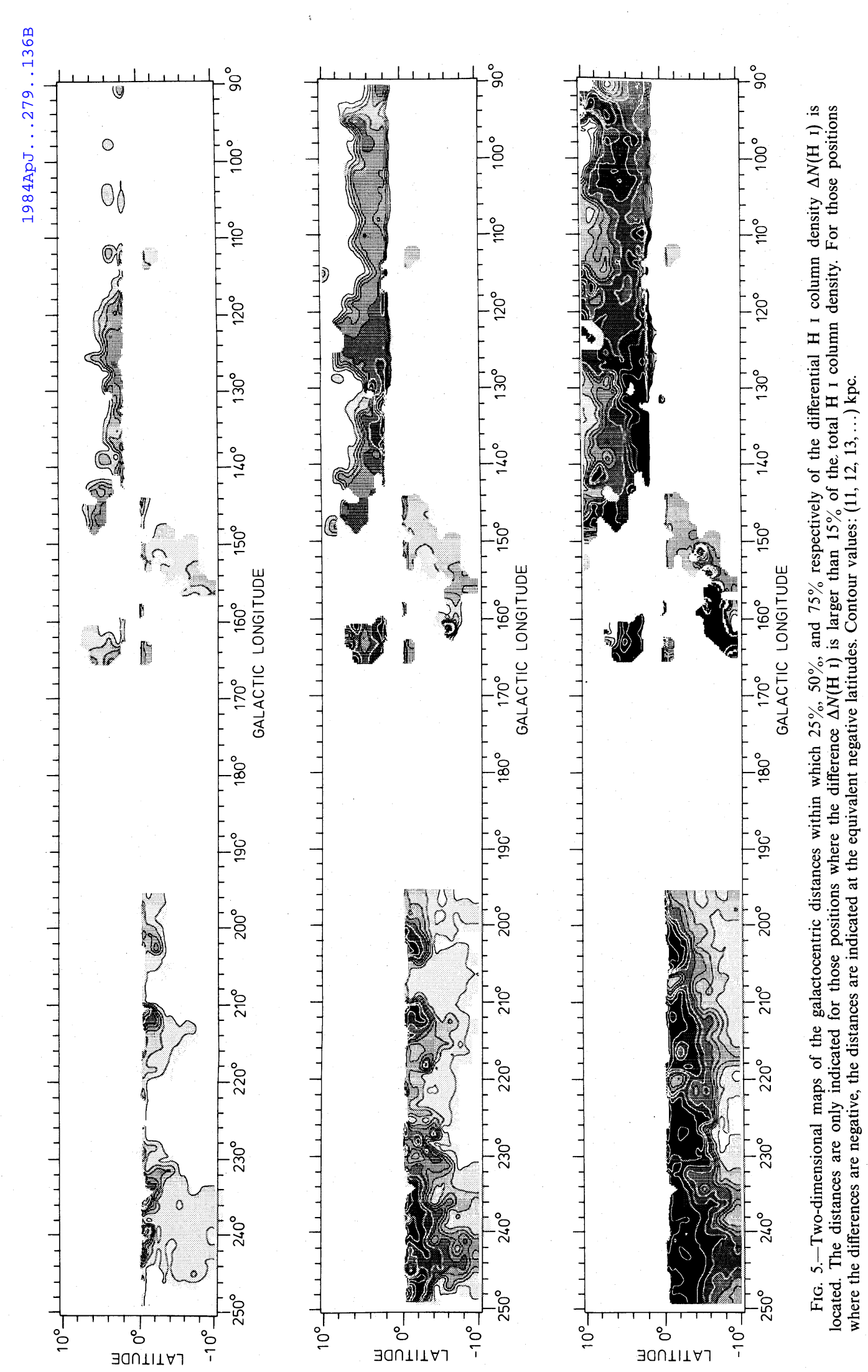


of the $\gamma$-ray emissivity $(70 \mathrm{MeV}-5 \mathrm{GeV}$ ) seems to be present in the outer Galaxy, the luminosity has been determined separately for $R<R_{\odot}$ (using the local emissivities; see Table 1 ) and for $R>R_{\odot}$ (using the emissivities determined in this paper).

Henderson, Jackson, and Kerr (1982) derived a total H I mass of the Galaxy of $4.8 \times 10^{9} M_{\odot}$ of which about $80 \%$ is situated beyond the solar circle. Using a conversion factor $N\left({ }^{13} \mathrm{CO}\right) / N\left(\mathrm{H}_{2}\right)=1 \times 10^{-6}$, Sanders derived a total $\mathrm{H}_{2}$ mass for $R<2 \mathrm{kpc}$ of $5 \times 10^{8} M_{\odot}$ and for $2 \mathrm{kpc}<R<R_{\odot}$ of $2.7 \times 10^{9} M_{\odot}$. The $\mathrm{H}_{2}$ mass for $R>R_{\odot}$ is derived by Kutner and Mead (1981) and can also be estimated from the results of Solomon, Stark, and Sanders (1983) (see § II). In both cases a value of $\sim 3 \times 10^{8} M_{\odot}$ is obtained. Dickman (1978), however, proposes an $N\left({ }^{13} \mathrm{CO}\right) / N\left(\mathrm{H}_{2}\right)$ conversion factor which is about 2.5 times larger, thus reducing the total $\mathrm{H}_{2}$ mass of the Milky Way by about a factor of 2.5 .

Using the uncertainties in the $\mathrm{H}_{2}$ mass mentioned above, a $10 \%$ uncertainty on the $\mathrm{H}$ I mass and the uncertainties in the $\gamma$-ray emissivities given in Table 1 , the $\gamma$-ray luminosity of the Milky Way is determined to be $(1.8-3.2) \times 10^{42}$ photons $\mathrm{s}^{-1}$ for the energy range $70 \mathrm{MeV}-5 \mathrm{GeV}$ and $(0.9-1.5) \times 10^{42}$ photons $\mathrm{s}^{-1}$ for the $150 \mathrm{MeV}-5 \mathrm{GeV}$ range, of which $\sim 50 \%$ originates outside the solar circle. An interpolation of the emissivities in the two energy ranges investigated yields a galactic luminosity of $(1.3-2.5) \times 10^{42}$ photons $\mathrm{s}^{-1}$ in the $100 \mathrm{MeV}-5 \mathrm{GeV}$ range, corresponding to $(1.6-3.2) \times 10^{39}$ ergs $\mathrm{s}^{-1}$ if an $E^{-1.8}$ power-law spectrum is assumed. These values are somewhat higher than the value $(1.2-1.5) \times 10^{42}$ photons $(>100 \mathrm{MeV}) \mathrm{s}^{-1}$ derived by Caraveo and Paul (1979) and $2 \times 10^{42}$ photons $(70 \mathrm{MeV}-5 \mathrm{GeV}) \mathrm{s}^{-1}$ derived by Mayer-Hasselwander et al. (1982) from unfoldings of the $S A S 2$ and $\operatorname{COS} B \gamma$-ray distributions, respectively.

\section{CONCLUSIONS}

For $R>R_{\odot}$ :

1. The $\gamma$-ray intensity is proportional to $N(\mathrm{H} \mathrm{I})$ only, and $N\left(\mathrm{H}_{2}\right)$ can be ignored to within the uncertainties of our analysis.

2. The $\gamma$-ray emissivity is independent of latitude to within $7 \%$, which implies an upper limit of $3 \times 10^{8} M_{\odot}$ to the mass of $\mathrm{H}_{2}$ in the outer Galaxy.

3. The $\gamma$-ray emissivity in the energy range $70 \mathrm{MeV}-5 \mathrm{GeV}$ is found to be only about $15 \%$ lower than the local value. If this result is statistically significant, it appears to be due to a gradient in the $70 \mathrm{MeV}-150 \mathrm{MeV}$ range $(\sim 30 \%)$.

4. About $25 \%$ of the $\gamma$-ray intensity originates from distances $14 \mathrm{kpc}<R<17 \mathrm{kpc}$.

5. The density of cosmic rays is large and nearly equal to the local value at distances up to about $17 \mathrm{kpc}$ from the galactic center. If the $30 \%$ decrease in the emissivity of the low-energy $\gamma$-rays is significant, then a gradient in the cosmic-ray electron density is required.

6. The $\gamma$-ray luminosity of the Milky Way is $(1.3-2.5) \times 10^{42}$ photons $\mathrm{s}^{-1}$ at energies above $100 \mathrm{MeV}$.

L. B. gratefully acknowledges support from the Netherlands Organization for the Advancement of Pure Research (ZWO), the General Research Board of the University of Maryland, and the NATO Scientific Affairs Division. The authors are indebted to their colleagues of the $\operatorname{COS} B$ collaboration for the use of unpublished data.

Blitz, L., Fich, M., and Kulkarni, S. R. 1983, Science, 220, 1233.

Blitz, L., Fich, M., and Stark, A. A. 1980, in IAU Symposium 87, Interstellar Molecules, ed. B. H. Andrew (Dordrecht: Reidel), p. 213.

-. 1982, Ap. J. Suppl., 49, 183.

Bloemen, J. B. G. M., Blitz, L., and Hermsen, W. 1983, in Southern Galactic

Surveys, ed. F. P. Israel and W. B. Burton (Dordrecht: Reidel), p. 89

Bloemen, J. B. G. M., et al. 1984, Astr. Ap., submitted.

Caraveo, P. A., and Paul, J. A. 1979, Astr. Ap., 75, 340.

Dickman, R. L. 1978, Ap. J. Suppl., 37, 407.

Fazio, G. G. 1967, Ann. Rev. Astr. Ap., 5, 481.

Fich, M., and Blitz, L. 1983, in Kinematics, Dynamics, and Structure of the Milky Way, ed. W. L. H. Shuter (Dordrecht: Reidel), p. 151.

Heiles, C., and Habing, H. J. 1974, Astr. Ap. Suppl., 14, 1.

Henderson, A. P., Jackson, P. D., and Kerr, F. J. 1982, Ap. J., 263, 116

Hermsen, W. 1980, Ph.D. thesis, University of Leiden, The Netherlands.

Kniffen, D. A., and Fichtel, C. E. 1981, Ap. J., 250, 389.

\section{REFERENCES}

Kulkarni, S. R., Blitz, L., and Heiles, C. 1982, Ap. J. (Letters), 259, L63.

Kutner, M. L., and Mead, K. 1981, Ap. J. (Letters), 249, L15.

Lebrun, F., et al. 1982, Astr. Ap., 107, 390

Mayer-Hasselwander, H. A., et al. 1982, Astr. Ap., 105, 164.

Sacher, W., and Schönfelder, V. 1983, Space Sci. Rev., 36, 249.

Sanders, D. B. 1981, Ph.D. thesis, State University of New York at Stony Brook.

Schlosser, W., and Feitzinger, J. V. 1983, Astr. Ap., 119, 42

Solomon, P. M., Stark, A. A., and Sanders, D. B. 1983, Ap. J. (Letters), 267, L29.

Stecker, F. W. 1971, Cosmic Gamma Rays (Baltimore: Mono Book Corporation).

Strong, A. W., et al. 1982, Astr. Ap., 115, 404.

Swanenburg, B. N., et al. 1981, Ap. J. (Letters), 243, L69.

Weaver, H., and Williams, D. R. W. 1973, Astr. Ap. Suppl., 8, 1.

Williams, D. R. W. 1973, Astr. Ap. Suppl., 8, 505.

Leo Blitz: Astronomy Program, University of Maryland, College Park, MD 20742

Hans Bloemen: Sterrewacht, Huygens Laboratorium, Wassenaarseweg 78, 2333 AL Leiden, The Netherlands

Wim HeRmSEN: Laboratory for Space Research Leiden, Wassenaarseweg 78, 2333 AL Leiden, The Netherlands 